\title{
Configurational partition functions and entropies for polyoxide chains having the repeat unit $\left[\left(\mathrm{CH}_{2}\right)_{y}-\mathrm{O}-\right]$
}

\author{
J. E. Mark ${ }^{\text {a) }}$ \\ Department of Chemistry and the Macromolecular Research Center, The University of Michigan, Ann \\ Arbor, Michigan 48109 \\ (Received 3 November 1976) \\ Rotational isomeric state theory is used to calculate values of the configurational partition function $z$ and \\ entropy $S$ for polyoxide chains having the repeat unit $\left[\left(\mathrm{CH}_{2}\right)_{y}-\mathrm{O}-\right]$. The values of $z$ are at least \\ qualitatively useful in interpreting the effect of the number $y$ of methylene groups in the repeat unit on the \\ characteristic ratio $\left\langle r^{2}\right\rangle_{0} / n I^{2}$ of the unperturbed dimensions relative to the number of skeletal bonds and \\ the average square of their lengths. For example, $z$ has a maximum (corresponding to maximum \\ "equilibrium flexibility" of the chain) at $y=3$, where $\left\langle r^{2}\right\rangle_{0} / n l^{2}$ is at a minimum. The configurational \\ entropy $S$, however, shows a very poor correlation with either $z$ or $\left\langle r^{2}\right\rangle_{0} / n l^{2}$; similar difficulties have been \\ noted in comparisons between some polypeptide chains, and between some polysaccharide chains.
}

\section{INTRODUCTION}

The class of chain molecules which has been most extensively studied ${ }^{1-4}$ with regard to its spatial configurations is the polyoxide series of repeat unit $\left[\left(\mathrm{CH}_{2}\right)_{y}-\mathrm{O}-\right]$. A number of members of this series have been characterized with regard to their configurationdependent properties, in particular their dimensions $\left\langle r^{2}\right\rangle_{0}$ as unperturbed by long-range interactions. ${ }^{1-5}$ More specifically, values of the unperturbed dimensions have been reported for the cases $y=1$ (polyoxymethylene), $y=2$ (polyoxyethylene), $y=3$ [poly(trimethylene oxide)], $y=4$ [poly(tetramethylene oxide)], $y=6[$ poly (hexamethylene oxide)], $y=10$ [poly (decamethylene oxide)], and $y=\infty$ (polyethylene). ${ }^{1-4}$ In order to facilitate comparisons between different polymers in this series, these results are generally expressed in terms of the characteristic ratio $\left\langle r^{2}\right\rangle_{0} / n l^{2}$ of the observed value of the unperturbed dimensions relative to the number of skeletal bonds in the chain and the average square of their lengths. These experimental values of the characteristic ratio, which pertain to $30{ }^{\circ} \mathrm{C},{ }^{2}$ are shown as a function of the number $y$ of methylene groups in the repeat unit, in the upper portion of Fig. 1. The most notable feature of these results is obviously the fact that $\left\langle r^{2}\right\rangle_{0} / n l^{2}$ goes through a pronounced minimum at $y=3$, thus identifying poly(trimethylene oxide) as the most "flexible" of the polyoxides, in the equilibrium sense of having the smallest spatial extension.

There are two rather different characteristics of a chain molecule which determine its spatial extension, as measured by its characteristic ratio. The first is thermodynamic and involves conformational preferences as exemplified by the energy differences between the rotational states accessible to the skeletal bonds making up the chain backbone. The second is geometric or structural, and involves skeletal bond angles and locations of rotational states; these determine the relative extension of the competing conformational alternatives. The exclusively thermodynamic contribution may be characterized in terms of the configurational partition function $Z$ of the chain or, alternatively, by means

\footnotetext{
2) Permanent address after July 1977: Department of Chemistry, University of Cincinnati, Cincinnati, $\mathrm{OH} 45221$.
}

of the configurational entropy $S$ derived therefrom. The purpose of the present study was therefore to use rotational isomeric state theory ${ }^{1}$ to calculate values of $Z$ and $S^{6-9}$ for members of the polyoxide series. Results thus obtained will establish the extent to which the unusual dependence of the characteristic ratio on the structure of the repeat unit is due to changes in $Z$ or $S$, which represent a purely thermodynamic measure of chain flexibility.

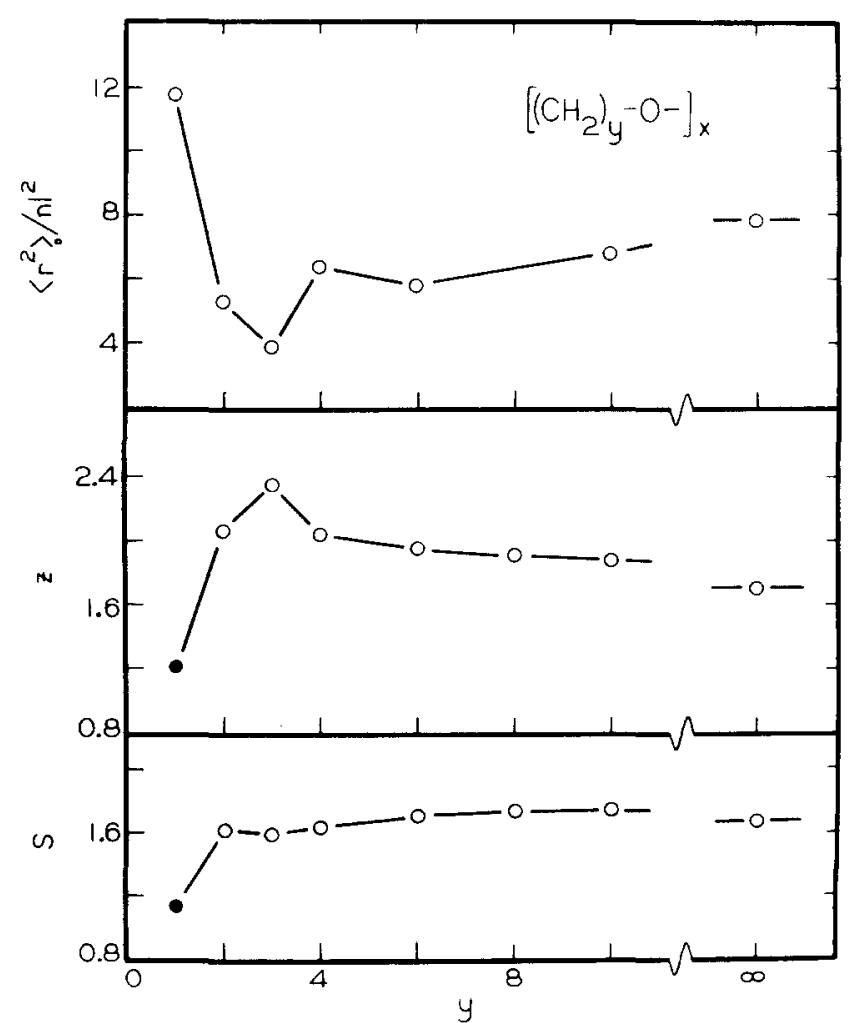

FIG. 1. Values of the characteristic ratio, and the configurational partition function and entropy for polyoxide chains shown as a function of the number $y$ of methylene groups in the polymer repeat unit. All results pertain to $30^{\circ} \mathrm{C}$, with those in the limit $y=\infty$ pertaining to polyethylene. The partition function $z$ is expressed per skeletal bond, and the entropy $S$ is given in units of cal deg-1 per mole of skeletal bonds. The points ( $\bullet$ ) representing the values of $z$ and $S$ for $y=1$ were calculated relative to the all-gauche conformation of the chain; all others (o) locate values of $z$ and $S$ calculated relative to the all-trans conformation. 


\section{THE ROTATIONAL ISOMERIC STATE MODEL}

The configurational partition function was calculated from the standard equation of rotational isomeric state theory ${ }^{1}$

$$
Z=\mathrm{J}^{*}\left(\prod_{i=1}^{n} \mathrm{U}_{i}\right) \mathrm{J},
$$

in which each $\mathrm{U}$ matrix contains the statistical weights for all conformations of a successive pair of skeletal bonds, and the pre- and postmultipliers extract the desired terms from the matrix product. In the polyoxides, there are nine such conformations for each pair of bonds, since the permitted rotational states are trans, gauche $e^{+}$, and gauche $e^{-}$located at rotational angles of $0^{\circ}$, $120^{\circ}$, and $-120^{\circ}$, respectively. Each of these statistical weights may be expressed as a Boltzmann factor in the energy of the associated conformation. The conformational energies required for this purpose have been established for the polyoxides of interest, both through the use of semiempirical potential energy functions, and by comparisons between theoretical and experimental values of configuration-dependent properties such as the unperturbed dimensions and dipole moments, and the temperature coefficients of these quantities. ${ }^{1,4}$ Although the best values of these conformational energies are reported elsewhere, ${ }^{4}$ it will be useful to summarize here the most important of these results. Gauche states which bring $\mathrm{CH}_{2}$ groups into proximity are of considerably higher energy than the corresponding trans states. When the skeletal bond in the gauche state is the $\mathrm{C}-\mathrm{C}$ bond, the energy difference is $0.5 \mathrm{kcal} \mathrm{mol}^{-1}$; when it is the shorter $\mathrm{C}-\mathrm{O}$ bond, the energy difference increases to approximately 0.9 $\mathrm{kcal} \mathrm{mol} \mathrm{m}^{-1}$. Gauche states which bring $\mathrm{O}$ atoms into proximity are approximately $0.4 \mathrm{kcal} \mathrm{mol}^{-1}$ lower in energy than the alternative trans states. The corresponding $\mathrm{CH}_{2} \cdots \mathrm{O}$ gauche interactions decrease the energy by $1.4 \mathrm{kcal} \mathrm{mol}^{-1}$ in the case of polyoxymethylene, and by $0.2-0.4 \mathrm{kcal} \mathrm{mol}^{-1}$ in the case of the other polyoxides. Finally, conformations giving rise to pentanetype interferences between two $\mathrm{CH}_{2}$ groups are completely excluded, while those involving two $O$ atoms or an $O$ atom and a $\mathrm{CH}_{2}$ group are only partially suppressed. ${ }^{4}$

Calculations were carried out for a range of temperatures centered on $30^{\circ} \mathrm{C}$. Since all skeletal bonds in the polyoxymethylene chain show a strong preference for gauche states, the regular all-gauche conformation $\left(g^{ \pm} g^{ \pm} g^{ \pm} g^{ \pm} ..\right)$) was chosen as the reference state for this molecule. In the case of the other polyoxides, at least some of the skeletal bonds exhibit a preference for trans states and the properties of these chains were therefore calculated relative to the all-trans conformation. Values of the configurational partition function per skeletal bond were calculated from the relationship

$$
z=Z^{1 / n} \text {. }
$$

Chains having at least $n=300$ skeletal bonds were employed in all calculations, thereby giving values of $z$ which are independent of $n$. The configurational entropy was calculated from the standard equation ${ }^{6,7,9}$

$$
S=R[\ln z+T(d \ln z / d T)]
$$

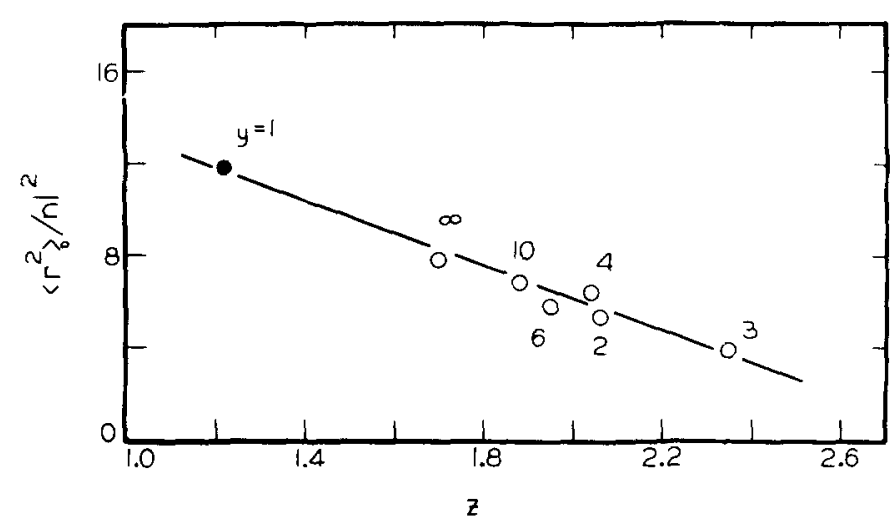

FIG. 2. The correlation between the characteristic ratio and the bond partition function; each point is identified by the number of methylene units in the repeat unit of the polyoxide being characterized.

and was expressed in cal $\mathrm{deg}^{-1}$ per mole of skeletal bonds.

\section{RESULTS AND DISCUSSION}

The central portion of Fig. 1 presents the calculated values of $z$ at $30^{\circ} \mathrm{C}$ as a function of the number $y$ of $\mathrm{CH}_{2}$ groups in the repeat unit. The bond partition function is seen to show the expected trends with $y$. It has its lowest value (corresponding to lowest flexibility) at $y=1$ (polyoxy methylene), whe re $\left\langle r^{2}\right\rangle_{0} / n l^{2}$ is a maximum. Similarly, its maximum value occurs at $y=3$ [poly (trimethylene oxide)], where the characteristic ratio has a minimum, at the unusually low value of $3.9 .^{2,3}$ In addition, as $y$ approaches the limit $y=\infty$ (polyethylene), $z$ asymptotically approaches a value which is very nearly the average of its maximum and minimum values, as does the characteristic ratio. Finally, these results explain why polyoxymethylene has a significantly higher value of $\left\langle r^{2}\right\rangle_{0} / n l^{2}$ than polyethylene. Although the preferred conformation in polyethylene is the alltrans form, ${ }^{1}$ which has the highest possible spatial extension, this structural feature of polyethylene is more than offset by the fact that conformationally, polyoxy methylene is a much more constrained molecule, as is indicated by its unusually small value of the bond partition function. The dependence of $z$ on $y$ is not as large as that shown by the characteristic ratio, but there is a good correlation between $\left\langle r^{2}\right\rangle_{0} / n l^{2}$ and $z$, as is shown in Fig. 2.

Values of $S$ at $30^{\circ} \mathrm{C}$ are shown in the lower portion of Fig. 1. This quantity is seen to have a very poor correlation with either $z$ or $\left\langle r^{2}\right\rangle_{0} / n l^{2}$; in fact, for $y$ $\geqslant 2, S$ is constant to within a few percent. At least in this class of chain molecules, increase in $z$ is accompanied by a decrease in its temperature coefficient $d \ln z / d T$; i.e., the more extensive the regions of configuration space already accessible to a skeletal bond, the smaller the fractional increase in these regions upon increase in temperature. As a consequence, $S$ as given by Eq. (3) is essentially a constant for almost all of the polyoxides. [A similar insensitivity of $S$ to structure occurs in the case of the polypeptides poly glycine and poly(L-alanine). These two chain molecules 
have essentially the same value of $S$ in spite of the fact that they have very different values of $\left.\left\langle r^{2}\right\rangle_{0} / n l^{2}.\right]^{6}$ More satisfactory results could possibly be obtained by replacing the discrete rotational states by regions or domains in the vicinity of each minimum in the conformational energy. ${ }^{6,10}$ Such refinement, however, would not change the basic difficulty that $z$ as well as $S$ lack the important geometric and structural information necessary for the quantitative characterization of chain flexibility and spatial extension.

Thus, although $z$ is obviously at least qualitatively useful in the case of polymers of closely related structure, such as the polyoxides studied here and various hydrocarbon and vinyl-type polymers, ${ }^{8}$ its general utility is quite limited, as is easily demonstrated by some illustrative cases. A hypothetical example would be two chain molecules with the same strong preference for trans states, one having equal skeletal bond angles, and the other unequal bond angles. These molecules would have essentially the same value of $z$ but their values of $\left\langle r^{2}\right\rangle_{0} / n l^{2}$ would be greatly different since the all-trans conformation corresponds to high chain extension in the former case, but to very small extension in the latter. ${ }^{1,11}$ The polysaccharides provide another example of limitations to the use of $z$ in character izing spatial extension. Amylose is a very inflexible chain in the sense that its accessible conformations are confined to a very small portion of configuration space. These accessible regions are well removed, however, from the regions corresponding to high extension and, as a result, amylose has a relatively small value of $\left\langle r^{2}\right\rangle_{0} / n l^{2}$. Its value is in fact smaller than that of cellulose, which is a less confined molecule, but one which has its preferred conformations in regions corresponding to high chain extension. ${ }^{8}$

It is thus obvious that while bond partition functions may be useful for qualitatively characterizing chain flexibility, at least within the same class of chain molecules, quantitative characterization generally requires the study of some configuration-dependent property, such as the unperturbed dimensions, which reflects the structural as well as thermodynamic features of the molecule.

It is a pleasure to acknowledge the partial financial support provided by the National Science Foundation, under Grant DMR 76-20036 (Polymers Program, Division of Materials Research).

${ }^{1} \mathrm{P}$. J. Flory, Statistical Mechanics of Chain Molecules (Interscience, New York, 1969).

${ }^{2}$ Y. Takahashi and J. E. Mark, J. Am. Chem. Soc. 98, 3756 (1976).

${ }^{3}$ D. S. Chiu, Y. Takahashi, and J. E. Mark, Polymer 17, 670 (1976).

${ }^{4}$ A. Abe and J. E. Mark, J. Am. Chem. Soc. 98, 6468 (1976).

${ }^{5}$ P. J. Flory, Principles of Polymer Chemistry (Cornell U. P., Ithaca, NY, 1953).

${ }^{6}$ D. A. Brant, W. G. Miller, and P. J. Flory, J. Mol. Biol. 23, 47 (1967).

${ }^{7}$ A. E. Tonelli, J. Chem. Phys. 52, 4749 (1970).

${ }^{8} \mathrm{P}$. J. Flory, Pure Appl. Chem. 26, 309 (1971).

${ }^{9}$ A. E. Tonelli, in Analytical Calorimetry, edited by R. S. Porter and J. F. Johnson (Plenum, New York, 1974), Vol. 3.

${ }^{10}$ A. E. Tonelli, Macromolecules 6, 503 (1973).

${ }^{11}$ J. E. Mark, J. Chem. Phys. 49, 1398 (1968). 